\title{
Comparative Study on Analysis and Design of Flat Slabs with Conventional Slabs
}

\author{
B. P. R. V. S. Priyatham, Er. D. V. S. K. Chaitanya
}

\begin{abstract}
Flat Slabs are highly adaptable elements widely used in construction, providing minimum depth, fast construction. In flat slabs, the beams used in conventional slabs are no more and the slab is made to rest directly over the columns. A drop panel or a column head is provided in case of higher loads to reduce the load intensity. Flat slabs are particularly suitable for areas where, for acoustic or fire reasons, tops of partitions need to be sealed to the slab soffit. This study concerns mainly the comparison of conventional and flat slab with and without slab drops by comparing the quantity of steel and bending moment in slabs of various spans $20 \times 20 \mathrm{~m}, 40 \times 40 \mathrm{~m}, 60 \times 60 \mathrm{~m}$. Each slab is sub divided into different panels of sizes $5 \times 5 \mathrm{~m}, 10 \times 10 \mathrm{~m}, 15 \times 15 \mathrm{~m}$.
\end{abstract}

Index Terms - Flat slab, Interior Panel, Exterior Panel, Column strip, Middle strip, Slab drop.

\section{INTRODUCTION}

Flat slabs construction system is the one in which the beams used in the conventional construction methods are eliminated. The slab rests directly on the column and the slab load is transferred directly to the columns and then to the foundation. The thickness of the slab near the support with the column is increased in order to support heavy loads and these are called slab drops and generally supplied with enlarged heads called column heads or capitals. Absence of beam gives a plain ceiling, thus giving better architectural appearance than in usual cases where beams are used.

Flat slab construction can significantly decrease floor to floor height, particularly in the lack of a false ceiling, since flat slab construction acts as a limiting factor in the positioning of horizontal services and partitions [1]. This can prove gainful in case of lower building height, decreased cladding expense and pre-fabricated services.

In case the client plans changes in the interior and wants to use the accommodation to suit the need, flat slab construction is the perfect choice as it offers that flexibility to the owner.[6]

\section{Types of Flat slabs:}

- Typical Flat Slab

- Slab without drop and column with column head

Manuscript revised July 19, 2019 and published on August 20, 2019 B. P. R.V.S. Priyatham, Department of Civil Engineering, GMR Institute of Technology, Rajam.

Er. D. V. S. K. Chaitanya, Department of Civil Engineering, ANU College of Engineering and Technology, Guntur.
- Slab with drop and column without column head

- Slab with drop and column with column head

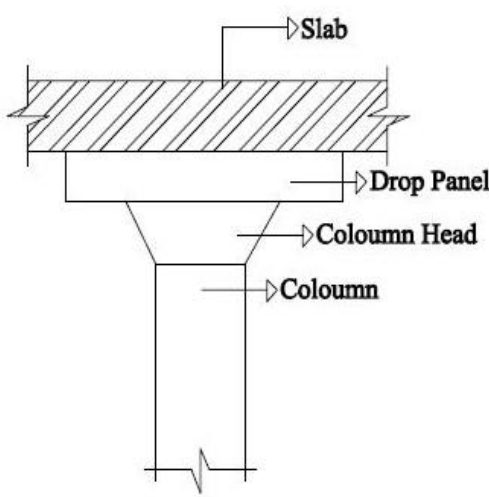

Fig.1 .Typical Components of a Flat Slab

Drop: The drops are nothing but the enlarged part below the slab at the intersection of column and slab. The main aim is to resist the punching shear which is predominant at the contact of slab and column Support. One of the most important criteria to be followed without fail is that the drop dimension should not be less than one -third of panel length in that direction.[2]

Column Head: Column Head is the bulged area over the column constructed to resist the slab load coming on to it. The column head is usually provided under the slab drop if necessary or as per the design requirement. Some amount of negative moment at the support is transferred from the slab to the column. The area at the support must be increased in order to resist this negative moment.

S.S.Patil, Rupali Sigi [7] said that in every code book it suggests any of the two methods as Direct Design Method and Equivalent Frame Method for gravity load analysis of flat slab. Design of Flat slab by Direct Design Method has some restrictions that (a) It should have minimum three spans in each directions. (b) It should not have staggered column orientation. Hence Equivalent Frame Method is adopted. 


\section{DESIGN METHODS AND METHODOLOGY}

The design of flat slab consists of two approximate methods namely Direct Design Method and Equivalent Frame Method.

- Step1: Finding the depth of slab.

- Step2: Finding the depth of slab drop.

- Step3: Finding the diameter of the column head.

- Step4: Finding the width of middle strip and column Strip.

- Step5: Consideration of the loads as per requirements.

(In the case of exterior panel calculation of the stiffness factor is compulsory)

- Step6: Calculation of the Factored Bending Moment.

- Step7: Calculation of negative and positive bending moments of column strip and middle strip.

- Step 8: calculation of shear stresses and check for the permissible shear.

- Step 9: calculation of reinforcements of all the column and middle strips.

- $\quad$ Step 10: check for the deflection.

\section{DESIGN CALCULATIONS}

In the present study we are comparing bending moments and area of steel for conventional slab, flat slab with drop and flat slab without drop having slab sizes of $20 \mathrm{~m} * 20 \mathrm{~m}$, $40 * 40 \mathrm{~m}, 60 \mathrm{~m} * 60 \mathrm{~m}$ each slab is further divided into panels of sizes $5 \mathrm{~m} * 5 \mathrm{~m}, 10 \mathrm{~m} * 10 \mathrm{~m}, 15 \mathrm{~m} * 15 \mathrm{~m}$ with exterior and interior panels.

The following are the considerations made in this design.

Dead load $=25 \mathrm{kN} / \mathrm{m}^{3}$

Live load $=4.5 \mathrm{kN} / \mathrm{m}^{2}$

Floor finish $=1.5 \mathrm{kN} / \mathrm{m}^{2}$

Column dia in case of flat slab with drop $=400 \mathrm{~mm}$

Column size in case of flat slab without drop = $300 \mathrm{~mm} * 450 \mathrm{~mm}$

Diameter of primary reinforcing bar $=16 \mathrm{~mm}, 10 \mathrm{~mm}$

Diameter of secondary reinforcing bar $=8 \mathrm{~mm}$

\section{CASE I: DESIGN OF FLAT SLAB WITHOUT SLAB DROP OF SIZE $20 \times 20 \mathrm{~m}$}

\section{Design of Interior Panel 5 x 5m}

Column size $=300 * 400 \mathrm{~mm}$

Live Load $=4.5 \mathrm{kN} / \mathrm{m}^{2}$

Floor Finish $=1.5 \mathrm{kN} / \mathrm{m}^{2}$

Height of column is $4 \mathrm{~m}$ above \& below the slab. $\mathrm{M}_{20} \& \mathrm{Fe}_{415}$

Depth of slab

$\mathrm{L} / \mathrm{d}=32$ (Clause 31.2)

$5000 / 32=\mathrm{d}$

$\mathrm{d}=156.25 \mathrm{~mm} \sim 160$

Provide Overall depth, $\mathrm{D}=185 \mathrm{~mm}$

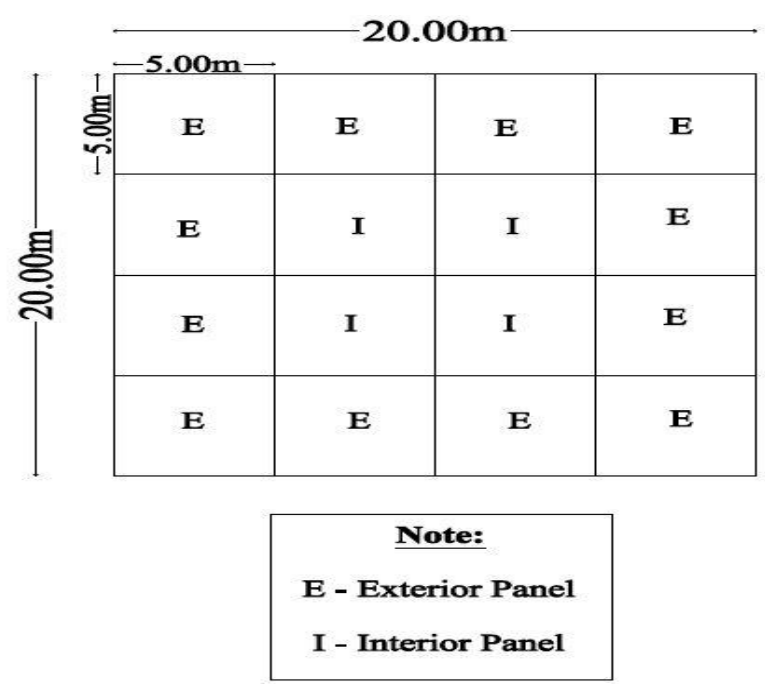

Fig.2 .Plan of Flat Slab without Slab Drop (20 x 20m)

\section{Load Calculations}

Self-weight of slab $=0.185 * 25=4.625 \mathrm{kN} / \mathrm{m}^{2}$

Floor Finish $=1.5 \mathrm{kN} / \mathrm{m}^{2}$

Live Load $=4.5 \mathrm{kN} / \mathrm{m}^{2}$

Total Factored $\mathrm{Load}=15.93 \mathrm{kN} / \mathrm{m}^{2}$

Dead Load $=4.625+1.5=6.125 \mathrm{kN} / \mathrm{m}^{2}$

Live Load $=4.5 \mathrm{kN} / \mathrm{m}^{2}$

\section{Along Longer direction}

For Slab

$\mathrm{K}_{\mathrm{S}}=4 \mathrm{EI} / \mathrm{l}=2.11 * 10^{6} \mathrm{E}$

For Column

$\mathrm{K}_{\mathrm{C}}=4 \mathrm{EI} / \mathrm{l}=1.6^{*} 10^{6} \mathrm{E}$

$\alpha_{c}=\Sigma K_{C} / \Sigma K_{S}=0.758$

\section{Along Shorter direction}

For Slab

$\mathrm{K}_{\mathrm{S}}=4 \mathrm{EI} / 1=2.11 * 10^{6} \mathrm{E}$

For Column

$\mathrm{K}_{\mathrm{C}}=4 \mathrm{EI} / 1=0.9 * 10^{6}$

$\alpha_{\mathrm{c}}=\Sigma \mathrm{K}_{\mathrm{C}} / \Sigma \mathrm{K}_{\mathrm{S}}=0.426$

Check for correction due to pattern loading

(IS 456-2000, Clause 31.4.6) [5]

Ratio $=$ Live Load $/$ Dead Load $=4.5 / 6.125=0.734>0.5$

Check for pattern loading is required

Check along longer direction

$1_{2} / l_{1}=5000 / 5000=1.0$

Live Load / Dead Load $=0.734$

$\mathrm{w}_{1} / \mathrm{w}_{\mathrm{d}}=0.5 ; \alpha_{\mathrm{cmin}}=0$

$\mathrm{w}_{1} / \mathrm{w}_{\mathrm{d}}=1.0 ; \alpha_{\mathrm{cmin}}=0.7$

$\alpha_{\text {Calculated }}>\alpha_{\text {cmin }}(0.9>0.7)$

No correction is required.

Check for pattern loading is same along shorter span also

Total design moment

Along Longer direction

$\mathrm{M}_{\mathrm{u}}=\mathrm{Wl}_{\mathrm{n}} / 8$

$\mathrm{W}=\mathrm{Wl}_{2} 1_{\mathrm{n}}$

$\mathrm{l}_{\mathrm{n}}=5-0.2-0.2=4.6 \mathrm{~m}$ 
$\mathrm{M}_{\mathrm{u}}=15.9375 * 5 * 4.6^{2} / 8$

$\mathrm{M}_{\mathrm{u}}=210.77 \mathrm{kNm}$

Along Shorter direction

$\mathrm{M}_{\mathrm{u}}=\mathrm{Wl}_{2} \mathrm{l}_{\mathrm{n}}^{2} / 8=210.77 \mathrm{kNm}$

Column Strip and Middle Strip

Along Longer direction (page no:53, Clause 1.31.1.1)

$2 * 0.25 * 5000=2500$

$2 * 0.25 * 5000=2500$

Provide Smaller of two values.

Width of column strip $=2500 \mathrm{~mm}$

Width of middle Strip $=5000-1250-1250=2500 \mathrm{~mm}$

Along Shorter direction

Width of Column strip $=2500 \mathrm{~mm}$

Middle Strip $=2500 \mathrm{~mm}$

Reinforcement in both directions

\section{Column Strip}

$\mathrm{M}_{\mathrm{u}}(-\mathrm{ve})=0.65 * 0.75 * 210.77=102.75 \mathrm{kNm}$

$\mathrm{A}_{\text {st }}(-\mathrm{ve})=1983.6 \mathrm{~mm}^{2}$

$\mathrm{M}_{\mathrm{u}}(+\mathrm{ve})=0.5 * 0.6 * 210.77=44.26 \mathrm{kNm}$

$\mathrm{A}_{\mathrm{st}}(+\mathrm{ve})=799.7 \mathrm{~mm}^{2}$

\section{Middle Strip}

$\mathrm{M}_{\mathrm{u}}(-\mathrm{ve})=(0.65 * 210.77)-102.75=34.25 \mathrm{kNm}$

$\mathrm{A}_{\mathrm{st}}(-\mathrm{ve})=612.6 \mathrm{~mm}^{2}$

$\mathrm{M}_{\mathrm{u}}(+\mathrm{ve})=(0.35 * 210.77)-102.75=28.81 \mathrm{kNm}$

$\mathrm{A}_{\mathrm{st}}(+\mathrm{ve})=512.9 \mathrm{~mm}^{2}$

Check for two-way shear

$\mathrm{d} / 2=160 / 2=80 \mathrm{~mm}$

$\mathrm{V}_{\mathrm{u}}=394.332 \mathrm{kN}$

$\mathrm{b}_{\mathrm{u}}=2(560+460)=2040 \mathrm{~mm}$

$\mathrm{T}_{\mathrm{v}}=\mathrm{V}_{\mathrm{u}} / \mathrm{b}_{\mathrm{u}} * \mathrm{~d}=1.208 \mathrm{~N} / \mathrm{mm}^{2} \quad$ IS 456:2000 (Clause 31.6.3.1)

$\mathrm{T}_{\mathrm{c}}{ }^{\prime}=\mathrm{K}_{\mathrm{s}} \mathrm{T}_{\mathrm{c}}, \mathrm{K}_{\mathrm{s}}=0.5+300 / 400=1.25>1.0$

$\mathrm{T}_{\mathrm{c}}=0.25 \sqrt{f c k}=1.11$

$\mathrm{T}_{\mathrm{c}}{ }^{\prime}=1.11 \mathrm{~N} / \mathrm{mm}^{2}, \mathrm{~T}_{\mathrm{v}}>\mathrm{T}_{\mathrm{c}}{ }^{\prime}$

Hence Shear Reinforcement is Required.

Design of shear Reinforcement

Consider $8 \phi$ Stirrups, 8 legged $\mathrm{Fe}_{415}$

$\mathrm{S}_{\mathrm{v}}=0.87 * 415 * \mathrm{~A}_{\mathrm{sv}} * \mathrm{~d} / \mathrm{V}_{\mathrm{us}}$

$\mathrm{A}_{\mathrm{sv}}=402 \mathrm{~m}^{2}$

$\mathrm{V}_{\mathrm{us}}=\mathrm{V}_{\mathrm{u}}-0.5 \mathrm{~T}_{\mathrm{c}} \mathrm{b}_{\mathrm{o}} \mathrm{dK} \mathrm{K}_{\mathrm{s}}=213.830 * 10^{3} \mathrm{~N}$

$\mathrm{S}_{\mathrm{v}}=108.93 \approx 110 \mathrm{~mm}$

Table I BENDING MOMENT AND AREA OF STEEL FOR INTERIOR PANEL WITHOUT DROP

\begin{tabular}{|c|c|c|c|c|}
\hline \multirow{2}{*}{$\begin{array}{c}\text { Panel } \\
\text { Sizes } \\
(\mathbf{m})\end{array}$} & \multicolumn{2}{|c|}{ Column Strip } & \multicolumn{2}{c|}{ Middle Strip } \\
\cline { 2 - 5 } & $\begin{array}{c}\text { Bending } \\
\text { Moment } \\
(\mathbf{k N m})\end{array}$ & $\begin{array}{c}\text { Area of } \\
\text { Steel } \\
\left(\mathbf{m m}^{\mathbf{2}}\right)\end{array}$ & $\begin{array}{c}\text { Bending } \\
\text { Moment } \\
(\mathbf{k N m})\end{array}$ & $\begin{array}{c}\text { Area of } \\
\text { Steel } \\
\left(\mathbf{m m}^{\mathbf{2}}\right)\end{array}$ \\
\hline \multirow{2}{*}{$\mathbf{5} * \mathbf{5}$} & $102.75(-\mathrm{ve})$ & 1983.6 & $34.25(-\mathrm{ve})$ & 612.65 \\
\cline { 2 - 5 } & $44.26(+\mathrm{ve})$ & 799.2 & $28.81(+\mathrm{ve})$ & 512.98 \\
\hline \multirow{2}{*}{$\mathbf{1 0} * \mathbf{1 0}$} & $1242.15(-\mathrm{ve})$ & 12927.8 & $414.18(-\mathrm{ve})$ & 3771.07 \\
\cline { 2 - 5 } & $535.48(+\mathrm{ve})$ & 4953.23 & $356.6(+\mathrm{ve})$ & 3319.53 \\
\hline \multirow{2}{*}{$\mathbf{1 5} * \mathbf{1 5}$} & $5406.85(-\mathrm{ve})$ & 42521.87 & $1802.28(-\mathrm{ve})$ & 11389.79 \\
\cline { 2 - 5 } & $2329.10(+\mathrm{ve})$ & 15068.89 & $1489.74(+\mathrm{ve})$ & 9291.62 \\
\hline
\end{tabular}

\section{Design of Exterior Panel 5 x 5m}

In the design of exterior panel preliminary data is same as of interior panel design and so the total design moments along the longer and shorter directions are considered from that and the moment distribution should be done. Also the width of column and middle strips is also same.

Total design moment $\mathrm{M}_{\mathrm{u}}=210.77 \mathrm{kNm}$

Moment Distribution

Interior negative design moment $=0.75-0.10 /\left(1+\frac{1}{\alpha c}\right)=$ 0.702

Positive design moment $=0.63-0.28 /\left(1+\frac{1}{\alpha c}\right)=0.497$

Exterior negative design moment $=0.65 /\left(1+\frac{1}{\alpha c}\right)=0.31$

Based on the moment distribution the area of steel can be calculated in both the directions and the check for two way shear is same as for interior panel.

Reinforcement in both directions

Column Strip

$\mathrm{M}_{\mathrm{u}}(-\mathrm{ve})=0.31 * 210.77=65.33 \mathrm{kNm}$

$\mathrm{A}_{\mathrm{st}}(-\mathrm{ve})=1207 \mathrm{~mm}^{2}$

$\mathrm{M}_{\mathrm{u}}(+\mathrm{ve})=0.6 * 0.497 * 210.77=62.85 \mathrm{kNm}$

$\mathrm{A}_{\mathrm{st}}(+\mathrm{ve})=1158.08 \mathrm{~mm}^{2}$

Middle Strip

$\mathrm{M}_{\mathrm{u}}(+\mathrm{ve})=(0.497 * 210.77)-61.37=41.92 \mathrm{kNm}$

$\mathrm{A}_{\mathrm{st}}(+\mathrm{ve})=755.6 \mathrm{~mm}^{2}$

TABLE II BENDING MOMENT AND AREA OF STEEL FOR EXTERIOR PANEL WITHOUT DROP

\begin{tabular}{|c|c|c|c|c|}
\hline \multirow[b]{2}{*}{$\begin{array}{c}\text { Panel } \\
\text { Sizes } \\
(\mathbf{m})\end{array}$} & \multicolumn{2}{|c|}{ Column Strip } & \multicolumn{2}{|c|}{ Middle Strip } \\
\hline & $\begin{array}{c}\text { Bending } \\
\text { Moment } \\
(\mathbf{k N m})\end{array}$ & $\begin{array}{c}\text { Area of } \\
\text { Steel } \\
\left(\mathrm{mm}^{2}\right)\end{array}$ & $\begin{array}{c}\text { Bending } \\
\text { Moment } \\
(\mathrm{kNm})\end{array}$ & $\begin{array}{c}\text { Area of } \\
\text { Steel } \\
\left(\mathrm{mm}^{2}\right)\end{array}$ \\
\hline \multirow{2}{*}{$5 * 5$} & $65.33(-\mathrm{ve})$ & 1207 & - & - \\
\hline & $62.85(+\mathrm{ve})$ & 1158.08 & 41.92(+ve) & 755.6 \\
\hline \multirow{2}{*}{$10 * 10$} & 163.12(-ve) & 1446.6 & - & - \\
\hline & $884.8(+\mathrm{ve})$ & 8583.4 & $618.7(+\mathrm{ve})$ & 5792.93 \\
\hline \multirow{2}{*}{$15 * 15$} & $262.85(-v e)$ & 1564.18 & - & - \\
\hline & $3501.3(+v e)$ & 24047.02 & $2859.41(+\mathrm{ve})$ & 18917.2 \\
\hline
\end{tabular}

\section{CASE II: DESIGN OF FLAT SLAB WITH SLAB DROP OF SIZE $20 \times 20 \mathrm{~m}$}

\section{Design of Interior Panel 5 x 5m}

Materials: M20 Grade and Fe415

Design of Interior panel dimension $5 \mathrm{~m} * 5 \mathrm{~m}$

$(1 / d)=32$

Two-way Continuous Slab as per IS 456: 2000

Thickness of slab at mid $\operatorname{span}(5000 / 32)=156.25 \approx 160 \mathrm{~mm}$

Adopt Effective Depth, $\mathrm{d}=160 \mathrm{~mm}$

Overall Depth, $\mathrm{D}=185 \mathrm{~mm}$ 


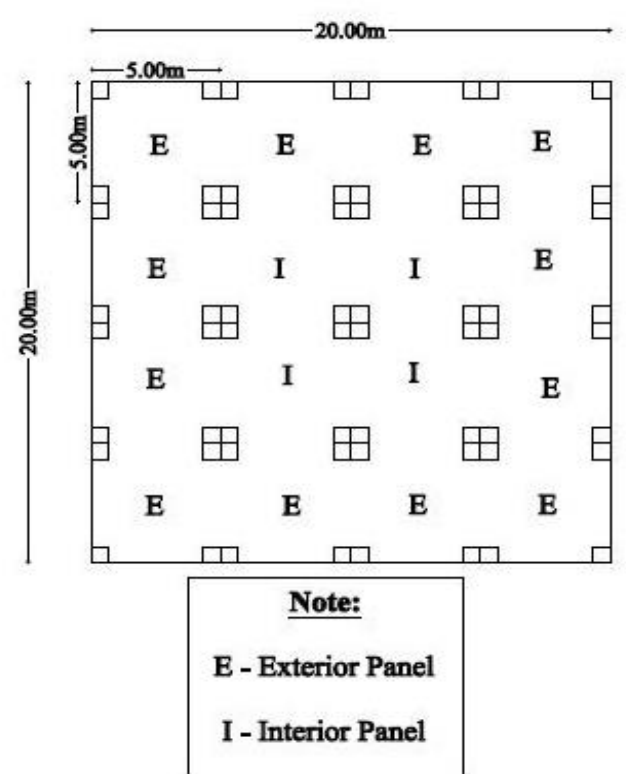

Fig.3 .Plan of Flat Slab with Slab Drop $(20$ x 20m)

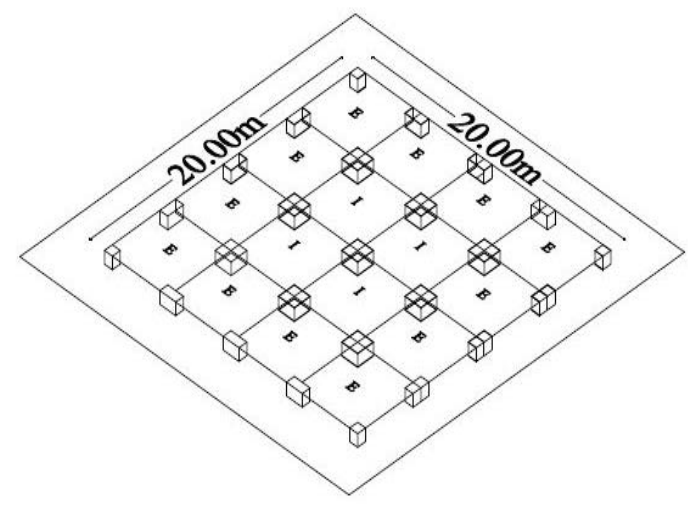

Fig.4 .Isometric View of Flat Slab with Slab Drop (20 x 20m)

According to Code ACI 318 the projection below slab drop should not be less than 1/4(Slab Thickness) and also should be less than or not equal to $100 \mathrm{~mm}$.

Hence thickness of slabs at drops $(185+100)=285 \mathrm{~mm}$

Column Head Diameter not greater than $0.25 \mathrm{~L}=0.25 * 5000$ $=1250 \mathrm{~mm}$

Length of Drop should not less than (L/3) $=5000 / 3=1666.6 \mathrm{~mm}$

Adopt Drop Width $=2500 \mathrm{~mm}$

Column Strip = Drop width $=2500 \mathrm{~mm}$

Width of middle Strip $=2500 \mathrm{~mm}$

\section{Loading Specifications}

Live Load of slab $=4.5 \mathrm{kN} / \mathrm{m}^{2}$

Dead Load of slab $=5.5625 \mathrm{kN} / \mathrm{m}^{2}$

Floor Finishers $=1.5 \mathrm{kN} / \mathrm{m}^{2}$

Total Service Load $=11.5625 \mathrm{kN} / \mathrm{m}^{2}$

Factored Load $=17.34 \mathrm{kN} / \mathrm{m}^{2}$

\section{Factored Bending Moment}

As per the IS 456-2000 Clause 31.4.2.2 the total moment is calculated as follows
$\mathrm{M}_{\mathrm{o}}=\mathrm{Wl}_{\mathrm{n}} / 8$

$\mathrm{L}_{\mathrm{n}}=5-1.25=3.75 \mathrm{~m}$

$\mathrm{L}_{1}=\mathrm{L}_{2}=5 \mathrm{~m}$

$\mathrm{W}=\mathrm{w}_{\mathrm{u}} \mathrm{L}_{2} \mathrm{l}_{\mathrm{n}}=325.125 \mathrm{kN}$

$\mathrm{M}_{\mathrm{o}}=152.40 \mathrm{kNm}$

Reinforcement

Column Strip

$\mathrm{M}_{\mathrm{u}}(-\mathrm{ve})=49 \% \mathrm{M}_{\mathrm{o}}=0.49 * 152.40=74.67 \mathrm{kNm}$

$\mathrm{M}_{\mathrm{u}}=0.89 * 415 * \mathrm{~A}_{\mathrm{st}} * \mathrm{~d} *\left(1-\mathrm{A}_{\mathrm{st}} \mathrm{f}_{\mathrm{y}} / \mathrm{b} * \mathrm{~d} * \mathrm{f}_{\mathrm{ck}}\right)$

$74.67 * 10^{6}=0.89 * 415 * \mathrm{~A}_{\mathrm{st}} * 260 *\left(1-415 \mathrm{~A}_{\mathrm{st}} / 2.5 * 10^{3} * 260 * 20\right)$

$\mathrm{A}_{\mathrm{st}}(-\mathrm{ve})=817.15 \mathrm{~mm}^{2}$

$\mathrm{M}_{\mathrm{u}}(+\mathrm{ve})=21 \% \mathrm{M}_{\mathrm{o}}=0.21 * 152.40=32 \mathrm{kNm}$

$\mathrm{A}_{\text {st }}(+\mathrm{ve})=344.89 \mathrm{~mm}^{2}$

\section{Middle Strip}

$\mathrm{M}_{\mathrm{u}}(-\mathrm{ve})=15 \% \mathrm{M}_{\mathrm{o}}=0.15^{*} 152.40=22.86 \mathrm{kNm}$

$\mathrm{A}_{\mathrm{st}}(-\mathrm{ve})=406 \mathrm{~mm}^{2}$

$\mathrm{M}_{\mathrm{u}}(+\mathrm{ve})=15 \% \mathrm{M}_{\mathrm{o}}=0.15^{*} 152.40=22.86 \mathrm{kNm}$

$\mathrm{A}_{\text {st }}(+\mathrm{ve})=406 \mathrm{~mm}^{2}$

Check For thickness of slab

For balanced section $\mathrm{M}_{\mathrm{u}}=0.138 \mathrm{f}_{\mathrm{ck}} \mathrm{bd}^{2}$

Thickness of slab near drops (Column strip)

$\mathrm{d}=\sqrt{\frac{74.6 * 10^{\wedge} 6}{0.138 * 20 * 2.5 * 10^{\wedge} 3}}=104.02 \mathrm{~mm}$

Thickness of slab in middle strips

$\mathrm{d}=\sqrt{\frac{22.86 * 10^{\wedge} 6}{0.138 * 20 * 2.5 * 10^{\wedge} 3}}=52.54 \mathrm{~mm}$

Overall depth near drops $=285 \mathrm{~mm}$

Effective depth near drop $=250 \mathrm{~mm}$

Overall depth (Middle Strip's) $=185 \mathrm{~mm}$

Effective depth (Middle Strip) $=160 \mathrm{~mm}$

Check for Shear Stress

Shear Stress is checked near the column head at section (D + d) near column head.

$\mathrm{W}_{1}=\Pi / 4(\mathrm{D}+\mathrm{d})^{2} \mathrm{Wu}=30.626 \mathrm{kN}$

Shear Force $=($ total load $)-($ Load on circular Area $)$

$$
=(17.34 * 5 * 5)-30.62=402.88 \mathrm{kN}
$$

Shear force per meter of perimeter $=[$ Shearforce $/ \pi(D+$ d) $]=85.537 \mathrm{kN}$

Shear Stress $=85.537 * 10^{3} /(1000 * 250)=0.34 \mathrm{~N} / \mathrm{mm}^{2}$

As per IS 456:2000 Clause 31.6.3.1

Permissible shear stress $=\mathrm{K}_{\mathrm{s}} \mathrm{T}_{\mathrm{c}}$

$\mathrm{K}_{\mathrm{s}}=\left(0.5+\boldsymbol{\beta}_{\mathrm{c}}\right), \boldsymbol{\beta}_{\mathrm{c}}=\mathrm{L} / \mathrm{L}_{2}=1$

$\mathrm{K}_{\mathrm{s}}=(0.5+1)=1.5$ But should not be greater than 1.0

$\mathrm{T}_{\mathrm{c}}=0.25 \sqrt{f c k}=0.25 \sqrt{20}=1.118 \mathrm{~N} / \mathrm{mm}^{2}$

$\mathrm{K}_{\mathrm{s}} \mathrm{T}_{\mathrm{c}}=1.118 \mathrm{~N} / \mathrm{mm}^{2}=0.342<1.118 \mathrm{~N} / \mathrm{mm}^{2}$

Check for Deflection Control

According to the IS 456:2000, Clause 23.2.1

$\mathrm{P}_{\mathrm{t}}=100 \mathrm{~A}_{\mathrm{st}} / \mathrm{bd}$

$$
=(100 * 406 / 1000 * 160)=0.254
$$

Figure:4 of IS 456:2000 modification factor for tension reinforcement

$\mathrm{K}_{\mathrm{t}}=1.8$

$(\mathrm{L} / \mathrm{d})_{\max }=(1.8 * 32)=57.6 / / 1.1 * 32=35.2$ 
$(\mathrm{L} / \mathrm{d})_{\text {provided }}=5000 / 160=31.25$

$31.25<57.6$

The designed Slab satisfies deflection limit.

Table III BENDING MOMENT AND AREA OF STEEL FOR INTERIOR PANEL WITH SLAB DROP

\begin{tabular}{|c|c|c|c|c|}
\hline \multirow[b]{2}{*}{$\begin{array}{c}\text { Panel } \\
\text { Sizes } \\
(\mathbf{m})\end{array}$} & \multicolumn{2}{|c|}{ Column Strip } & \multicolumn{2}{|c|}{ Middle Strip } \\
\hline & $\begin{array}{c}\text { Bending } \\
\text { Moment } \\
(\mathrm{kNm})\end{array}$ & $\begin{array}{c}\text { Area of } \\
\text { Steel } \\
\left(\mathrm{mm}^{2}\right)\end{array}$ & $\begin{array}{c}\text { Bending } \\
\text { Moment } \\
(\mathbf{k N m})\end{array}$ & $\begin{array}{c}\text { Area of } \\
\text { Steel } \\
\left(\mathrm{mm}^{2}\right)\end{array}$ \\
\hline \multirow{2}{*}{$5 * 5$} & 74.67(-ve) & 817.15 & $22.86(-\mathrm{ve})$ & 406 \\
\hline & $32(+v e)$ & 344.89 & $22.86(+v e)$ & 406 \\
\hline \multirow{2}{*}{$10 * 10$} & $826.87(-v e)$ & 5786.4 & 253.12(-ve) & 2258.09 \\
\hline & $354.37(+\mathrm{ve})$ & 2394.76 & $253.12(+v e)$ & 2258.09 \\
\hline \multirow{2}{*}{$15 * 15$} & $5703.49(-v e)$ & 33020 & $1745.96(-\mathrm{ve})$ & 8869.95 \\
\hline & $2444.35(+v e)$ & 12661.14 & $1745.96(+v e)$ & 8869.95 \\
\hline
\end{tabular}

\section{Design of Exterior Panel 5 x 5m}

Loading class $=4.5 \mathrm{kN} / \mathrm{m}^{2}, \mathrm{M} 20, \mathrm{Fe} 415$

Height of Story $=3 \mathrm{~m}$

Thickness of slab in column strip $=285 \mathrm{~m}$

Thickness of slab in middle strip $=185 \mathrm{~mm}$

Dimensions of Flat slab

Width of middle strip $=$ Width of column $=$

Drop width $=2.5 \mathrm{~m}$

\section{Stiffness Computation}

$\mathrm{K}_{\mathrm{c}}=4 \mathrm{E}_{\mathrm{c}} \mathrm{l}_{\mathrm{c}} / \mathrm{l}=1.67 * 10^{6} \mathrm{E}_{\mathrm{c}}$

Assuming columns both at top and bottom

$\mathrm{K}_{\mathrm{c}}=2\left(1.67 * 10^{6}\right) \mathrm{E}_{\mathrm{c}}$

Stiffness of slab

$\mathrm{K}_{\mathrm{s}}=4 * 5000 * 300^{3} / 12 * 5000=21.33 * 10^{6} \mathrm{E}_{\mathrm{c}}$

$\boldsymbol{\alpha}_{\mathrm{c}}=\boldsymbol{\Sigma} \mathrm{K}_{\mathrm{c}} / \boldsymbol{\Sigma} \mathrm{K}_{\mathrm{s}}=0.156$

$\boldsymbol{\alpha}_{\mathrm{c}}(\min )=0.7$ for $\left(\mathrm{L}_{2} / \mathrm{L}_{1}\right)=1.0$

$\left(1+1 / \boldsymbol{\alpha}_{\mathrm{c}}\right)=7.41$

$\mathrm{L}_{\mathrm{n}}=5-1.25=3.75 \mathrm{~m}$

Bending Moment

Total Load $=\mathrm{W}=\mathrm{w}_{\mathrm{u}} \mathrm{l}_{2} \mathrm{~L}_{\mathrm{n}}=325.125 \mathrm{kN}$

Bending Moment $\mathrm{M}_{\mathrm{o}}=(325.125 * 3.75) / 8=152.40 \mathrm{kNm}$

Interior Negative design moment

$\left(0.75-\frac{0.10}{1+1 / \alpha c}\right) \mathrm{M}_{\mathrm{o}}=112.24 \mathrm{kNm}$

Positive design moment

$\left(0.63-\frac{0.28}{1+1 / \alpha c}\right) \mathrm{M}_{\mathrm{o}}=78.45 \mathrm{kNm}$

Exterior Negative design moment

$\left(\frac{0.65}{1+1 / \alpha c}\right) \mathrm{M}_{\mathrm{o}}=13.36 \mathrm{kNm}$

Interior Negative design moment

Column strip $=0.75 * 112.24=84.18 \mathrm{kNm}$

Middle strip $=0.25 * 112.24=28.06 \mathrm{kNm}$

Exterior Negative design moment

Column strip $=13.36 \mathrm{kNm}$

Middle Strip $=0$

Positive moment in column strip

$0.60 * 84.18=50.508 \mathrm{kNm}$
Positive moment in Middle strip

$0.40 * 84.18=33.672 \mathrm{kNm}$

Thickness of Slab near Drops

$\mathrm{d}=\sqrt{M u / 0.138 f c k b}=110.45 \mathrm{~mm}$

Thickness of Slab in middle Strip

$\mathrm{d}=\sqrt{M u / 0.138 f c k b}=69.85 \mathrm{~mm}$

Reinforcement

Column Strip Interior Negative Bending moment

$=0.87 \mathrm{f}_{\mathrm{y}} \mathrm{A}_{\mathrm{st}} \mathrm{d}\left(1-\frac{\text { Astfy }}{b d \mathrm{fck}}\right)$

$84.18 * 10^{6}=0.87 * 415 * \mathrm{~A}_{\mathrm{st}} * 260 *\left(1-\frac{415 \text { Ast }}{2.5 * 10^{3} * 260 * 20}\right)$

$\mathrm{A}_{\mathrm{st}}=924.47 \mathrm{~mm}^{2}$

$A_{\text {st }}$ for positive Bending Moment

$50.508 * 10^{6}=0.87 * 415 * \mathrm{~A}_{\mathrm{st}} * 160 *\left(1-\frac{415 \text { Ast }}{2500 * 160 * 20}\right)$

$\mathrm{A}_{\mathrm{st}}=918.52 \mathrm{~mm}^{2}$

Middle Strip

$33.672 * 10^{6}=0.87 * 415 * \mathrm{~A}_{\mathrm{st}} * 160 *\left(1-\frac{415 \text { Ast }}{2500 * 160 * 20}\right)$

$\mathrm{A}_{\mathrm{st}}=601.97 \mathrm{~mm}^{2}$

Column Strip

$13.36 * 10^{6}=0.87 * 415 * \mathrm{~A}_{\mathrm{st}} * 260 *\left(1-\frac{415 \text { Ast }}{2500 * 260 * 20}\right)$

$\mathrm{A}_{\mathrm{st}}=143.04 \mathrm{~mm}^{2}$

TABLE IV BENDING MOMENT AND AREA OF STEEL FOR EXTERIOR PANEL WITH SLAB DROP

\begin{tabular}{|c|c|c|c|c|}
\hline \multirow[b]{2}{*}{$\begin{array}{c}\text { Panel } \\
\text { Sizes } \\
(\mathbf{m})\end{array}$} & \multicolumn{2}{|c|}{ Column Strip } & \multicolumn{2}{|c|}{ Middle Strip } \\
\hline & $\begin{array}{c}\text { Bending } \\
\text { Moment } \\
(\mathbf{k N m})\end{array}$ & $\begin{array}{c}\text { Area of } \\
\text { Steel } \\
\left(\mathrm{mm}^{2}\right)\end{array}$ & $\begin{array}{c}\text { Bending } \\
\text { Moment } \\
(\mathrm{kNm})\end{array}$ & $\begin{array}{c}\begin{array}{c}\text { Area of } \\
\text { Steel } \\
\left(\mathrm{mm}^{2}\right)\end{array} \\
\end{array}$ \\
\hline \multirow{2}{*}{$5 * 5$} & $84.18(-v e)$ & 924.47 & - & - \\
\hline & $50.5(+\mathrm{ve})$ & 918.52 & $33.67(+v e)$ & 601.97 \\
\hline \multirow{2}{*}{$10 * 10$} & 932.13(-ve) & 6577 & - & - \\
\hline & $559.28(+\mathrm{ve})$ & 5192 & $372.85(+v e)$ & 3376.65 \\
\hline \multirow{2}{*}{$15 * 15$} & 6429.5(-ve) & 6577 & - & - \\
\hline & $3857.7(+\mathrm{ve})$ & 5192 & $2571.82(+\mathrm{ve})$ & 3376.65 \\
\hline
\end{tabular}

CASE III: DESIGN OF CONVENTIONAL TWO WAY SLAB OF SIZE $20 \times 20 \mathrm{~m}$

Materials: Adopt M20\&Fe415.

$\mathrm{L}_{\mathrm{x}}=5 \mathrm{~m}$

$\mathrm{L}_{\mathrm{y}}=5 \mathrm{~m}\left(\mathrm{~L}_{\mathrm{y}} / \mathrm{L}_{\mathrm{x}}=1\right)$

$\mathrm{F}_{\mathrm{ck}}=20 \mathrm{~N} / \mathrm{mm}^{2}$

$\mathrm{F}_{\mathrm{y}}=415 \mathrm{~N} / \mathrm{mm}^{2}$

Depth of slab

As the span more than $3.5 \mathrm{~m}$ adopt a span/depth ratio of 25

Depth $=$ Span $/ 25=5000 / 25=200 \mathrm{~mm}$

Adopt effective depth, $d=175 \mathrm{~mm}$

Effective Span

Effective span $=$ Clear span + Effective depth $=4.87 \mathrm{~m}$

Loads

Self-weight of slab $=(0.2 * 2.5)=5 \mathrm{kN} / \mathrm{m}^{2}$

Live load on slab $=4 \mathrm{kN} / \mathrm{m}^{2}$

Floor finish $=1.5 \mathrm{kN} / \mathrm{m}^{2}$ 
Total working load $=10.5 \mathrm{kN} / \mathrm{m}^{2}$

Design ultimate load $=15.75 \mathrm{kN} / \mathrm{m}^{2}$

i) Interior Panel

Ultimate Design moments \& Shear forces

For, $\left(\mathrm{L}_{\mathrm{y}} / \mathrm{L}_{\mathrm{x}}=1\right) \alpha_{\mathrm{x}}=0.032, \alpha_{\mathrm{y}}=0.024$

$\mathrm{M}_{\mathrm{ux}}=\alpha_{\mathrm{x}} \mathrm{W}_{\mathrm{u}} \mathrm{L}_{\mathrm{x}}^{2}=11.95 \mathrm{kNm}$

$\mathrm{M}_{\mathrm{uy}}=\alpha_{\mathrm{y}} \mathrm{w}_{\mathrm{u}} \mathrm{L}_{\mathrm{y}}^{2}=8.96 \mathrm{kNm}$

$\mathrm{V}_{\mathrm{ux}}=0.5 \mathrm{w}_{\mathrm{u}} \mathrm{L}_{\mathrm{x}}=32.60 \mathrm{kNm}$

Area of reinforcement

$\mathrm{A}_{\mathrm{st}}=0.5 \mathrm{f}_{\mathrm{ck}} / \mathrm{f}_{\mathrm{y}}\left[1-\sqrt{1-\frac{4.6 M u}{f c k b d^{\wedge} 2}}\right] \mathrm{bd}=193.67 \mathrm{~mm}^{2}$

$\mathrm{A}_{\mathrm{st}} \min =0.0012 * 175 * 1000=210 \mathrm{~mm}^{2}$

$\mathrm{A}_{\mathrm{st}}<\mathrm{A}_{\mathrm{st}} \min$

Hence provide minimum reinforcement

Reinforcements Along Short span

$\mathrm{A}_{\mathrm{st}}=0.5 \mathrm{f}_{\mathrm{ck}} / \mathrm{f}_{\mathrm{y}}\left[1-\sqrt{1-\frac{4.6 M u}{f c k b d^{\wedge} 2}}\right] \mathrm{bd}=145 \mathrm{~mm}^{2}$

$\mathrm{A}_{\mathrm{st}} \min =210 \mathrm{~mm}^{2}$

Hence provide minimum reinforcement

ii) Two Adjacent Edges Discontinuous

Ultimate Design moments \& Shear forces

As per the codebook the ratio $\left(\mathrm{L}_{\mathrm{y}} / \mathrm{L}_{\mathrm{x}}=1\right)$

$\mathrm{M}_{\mathrm{ux}(-\mathrm{ve})}=\left(\alpha_{\mathrm{x}} \mathrm{w}_{\mathrm{u}} \mathrm{L}_{\mathrm{x}}^{2}\right)=0.047 * 15.75 * 4.87^{2}=17.55 \mathrm{kNm}$

$\mathrm{M}_{\mathrm{ux}(+\mathrm{ve})}=\left(\alpha_{\mathrm{x}} \mathrm{w}_{\mathrm{u}} \mathrm{L}_{\mathrm{y}}{ }^{2}\right)=0.035 * 15.75 * 4.87^{2}=13.07 \mathrm{kNm}$

$\mathrm{M}_{\text {uy(-ve })}=\left(\alpha_{\mathrm{x}} \mathrm{w}_{\mathrm{u}} \mathrm{L}_{\mathrm{y}}{ }^{2}\right)=0.042 * 15.75 * 4.87^{2}=15.668 \mathrm{kNm}$

$\mathrm{M}_{\text {uy }(+\mathrm{ve})}=\left(\alpha_{\mathrm{x}} \mathrm{W}_{\mathrm{u}} \mathrm{L}_{\mathrm{y}}{ }^{2}\right)=0.035 * 15.75 * 4.87^{2}=13.07 \mathrm{kNm}$

Area of reinforcement

$\mathrm{A}_{\mathrm{st}}(-\mathrm{ve})=0.5 \mathrm{f}_{\mathrm{ck}} / \mathrm{f}_{\mathrm{y}}\left[1-\sqrt{1-\frac{4.6 M u}{f c k b d^{\wedge} 2}}\right] \mathrm{bd}=287.7 \mathrm{~mm}^{2}$

Reinforcement along Short span

$\mathrm{A}_{\mathrm{st}}(-\mathrm{ve})=255.668 \mathrm{~mm}^{2}$

$\mathrm{A}_{\mathrm{st}}(+\mathrm{ve})=212 \mathrm{~mm}^{2}$

iii) One Edge Discontinuous

Ultimate Design moments \& Shear forces

For, $\left(\mathrm{L}_{\mathrm{y}} / \mathrm{L}_{\mathrm{x}}=1\right) \alpha_{\mathrm{x}}=0.047, \alpha_{\mathrm{y}}=0.035$

$\mathrm{M}_{\mathrm{ux}(-\mathrm{ve})}=\alpha_{\mathrm{x}} \mathrm{W}_{\mathrm{u}} \mathrm{L}_{\mathrm{x}}{ }^{2}=0.037 * 15.75^{*} 4.87^{2}=13.82 \mathrm{kNm}$

$\mathrm{M}_{\mathrm{ux}(+\mathrm{ve})}=\alpha_{\mathrm{x}} \mathrm{W}_{\mathrm{u}} \mathrm{L}_{\mathrm{y}}{ }^{2}=0.028 * 15.75^{*} 4.87^{2}=13.07 \mathrm{kNm}$

$\mathrm{M}_{\text {uy(-ve) }}=\alpha_{\mathrm{x}} \mathrm{w}_{\mathrm{u}} \mathrm{L}_{\mathrm{y}}{ }^{2}=0.037 * 15.75 * 4.87^{2}=15.668 \mathrm{kNm}$

$\mathrm{M}_{\mathrm{uy}(+\mathrm{ve})}=\alpha_{\mathrm{x}} \mathrm{W}_{\mathrm{u}} \mathrm{L}_{\mathrm{y}}{ }^{2}=0.028 * 15.75^{*} 4.87^{2}=13.07 \mathrm{kNm}$

$\mathrm{V}_{\mathrm{ux}}=0.5 \mathrm{w}_{\mathrm{u}} \mathrm{L}_{\mathrm{x}}=0.5 * 15.75 * 4.87=38.35 \mathrm{kNm}$

Area of reinforcement

$\mathrm{A}_{\mathrm{st}}(-\mathrm{ve})=0.5 \mathrm{f}_{\mathrm{ck}} / \mathrm{f}_{\mathrm{y}}\left[1-\sqrt{1-\frac{4.6 M u}{f c k b d^{\wedge} 2}}\right] \mathrm{bd}=224.8 \mathrm{~mm}^{2}$

$\mathrm{A}_{\text {st }}(+\mathrm{ve})=212 \mathrm{~mm}^{2}$

\section{Reinforcement Along Short span}

$\mathrm{A}_{\mathrm{st}}(-\mathrm{ve})=224.8 \mathrm{~mm}^{2}$

$\mathrm{A}_{\mathrm{st}}(+\mathrm{ve})=212 \mathrm{~mm}^{2}$

Check for shear stresses

$\mathrm{Tv}=\mathrm{Vu} / \mathrm{bd}=0.186 \mathrm{~N} / \mathrm{mm}^{2}$

$\mathrm{P}_{\mathrm{t}}=100 \mathrm{~A}_{\mathrm{st}} / \mathrm{bd}=0.135 \mathrm{~N} / \mathrm{mm}^{2}$

From IS 456 Table $19, \mathrm{Tc}=0.28 \mathrm{~N} / \mathrm{mm}^{2}$

$\mathrm{Tv}<\mathrm{Tc}$
Hence no shear reinforcement is required.

Check for deflection

As per IS 456:2000 the ratio of $(\mathrm{L} / \mathrm{d})_{\text {basic }}=20$

$\mathrm{P}_{\mathrm{t}=0.135}$

$\mathrm{K}_{\mathrm{t}=1.8}$

$(\mathrm{L} / \mathrm{d})_{\max }=20 * 1.8$

$(\mathrm{L} / \mathrm{d})_{\text {provided }}=5000 / 170=28.5<36$

Hence deflection control is satisfied

Check for cracking

Steel provided is more than the minimum \% of reinforcement Spacing of main steel $<3 \mathrm{~d}=3 * 175=525 \mathrm{~mm}$

Diameter of reinforcement $<\mathrm{D} / 8=200 / 8=25 \mathrm{~mm}$

Hence cracks are within permissible limits.

Torsion reinforcement at corners

Area of reinforcement in each 4 layers $=(0.25 * 210)=$ $157.5 \mathrm{~mm}^{2}$

Distance over which torsion reinforcement is provided $=$ $1 / 5($ short span $)=1 / 5(50000)=1000 \mathrm{~mm}$

Provide $6 \mathrm{~mm}$ diameter at $1000 \mathrm{~mm}$ centers for a length of 1000mm@all 4 layers in 4 corners.

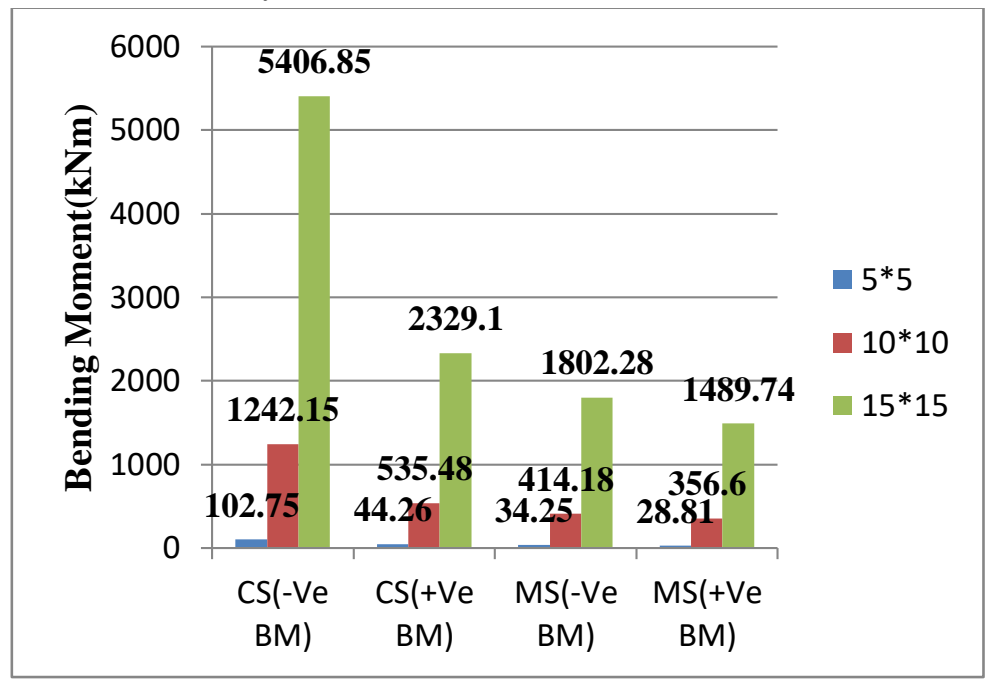

Fig.5 .Variation of Interior Panel Bending Moments for different panel sizes for flat slab without drop

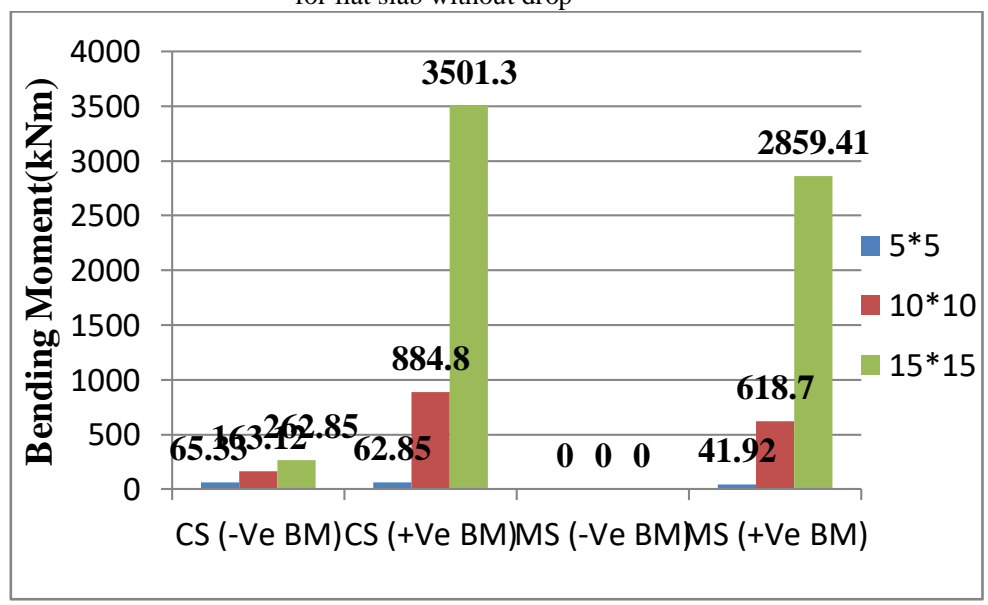

Fig.6 .Variation of Exterior Panel Bending Moments for different panel sizes for flat slab without drop 


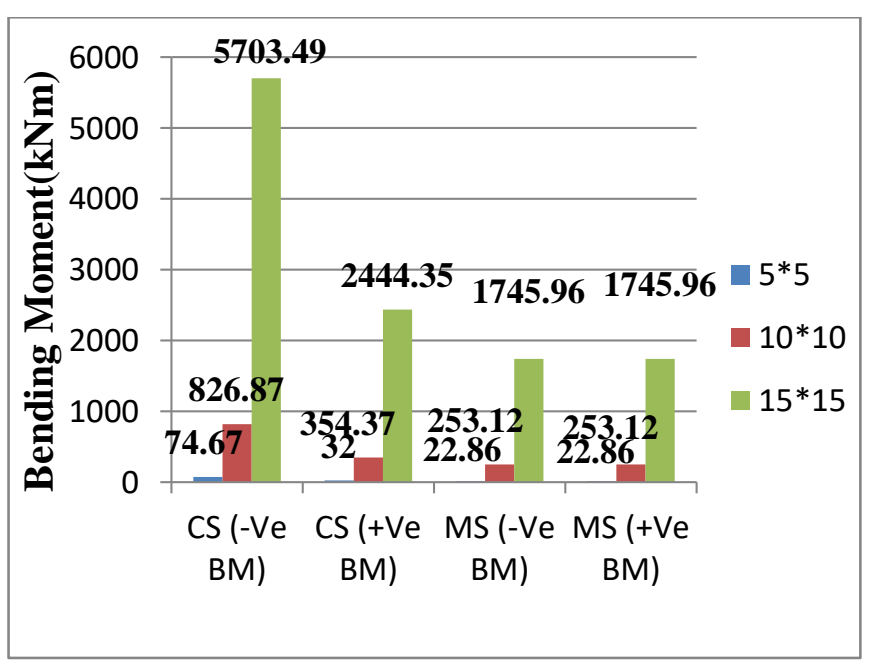

Fig.7 .Variation of Interior Panel Bending Moments for different panel sizes for flat slab with Slab Drop

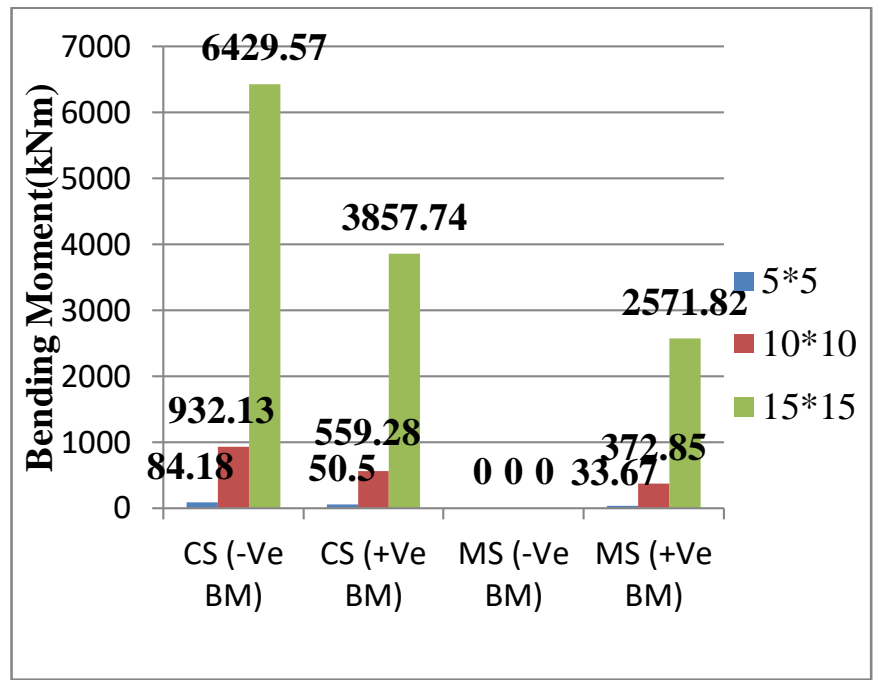

Fig.8 .Variation of Exterior Panel Bending Moments for different panel sizes for flat slab with Slab Drop

\section{Conclusion}

1. It was observed that the value of the bending moment of flat slab without drop increases when compared to flat slab with drop of equal dimensions.

2. It was observed that the value of the bending moment of flat slab is higher when compared to conventional slab of equal dimensions.

\section{AUTHORS PROFILE}

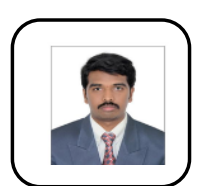

B. P. R. V. S. Priyatham is an Assistant Professor at GMR Institute of Technology, Rajam. He has graduated in Masters of Engineering from Andhra University and his research area is Coldformed Steel Structures.

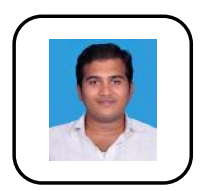

Er D. V. S. K. Chaitanya is an Assistant Professor at ANU College of Engineering and Technology, Acharya Nagarjuna University, Guntur. He has graduated in Masters of Engineering from Andhra University and is currently pursuing his $\mathrm{PhD}$ in Civil Engineering. $\mathrm{He}$ is a member of professional bodies like ICI and IIID.
3. When a flat Slab with drop is considered it was observed that there is an increase in the percentage of bending moment of $44.65,54.42$ for panel dimensions of $5 * 5 \mathrm{~m}$, $10 * 10 \mathrm{~m}$ respectively when compared with flat slab without drop and there is a decrease in the percentage of bending moment of 3.90 for panel dimensions $15 * 15 \mathrm{~m}$.

4. When a flat Slab with drop is considered it was observed that there is an increase in the percentage of bending moment of $56.45,67.06$ for panel dimensions of $5 * 5 \mathrm{~m}$, $10 * 10 \mathrm{~m}$ respectively when compared with flat slab without drop and there is a decrease in the percentage of bending moment of 4.70 for panel dimensions $15^{*} 15 \mathrm{~m}$.

5. When a flat Slab with drop is considered it was observed that there is a decrease in the percentage of bending moment of $18.97,82.50$, and 95.91 for panel dimensions of $5 * 5 \mathrm{~m}, 10 * 10 \mathrm{~m}$, and $15 \mathrm{~m} * 15 \mathrm{~m}$ respectively.

\section{REFERENCES}

[1] Gaurav Ravindran Chavan, "Analysis and Design of Flat Slab," International Journal of Latest Trends in Engineering and Technology, vol.7, Issue 1, 2016.

[2] Patil, K.S.,Gore, N.G.,Salunke, P.J., "Optimum design of reinforced concrete flat slab with Drop Panel", International Journal of Recent Technology and Engineering, Vol. 2, September 2013.

[3] Bhavikatti S. S, Advance R.C.C. Design, New Age International (P) Limited Publishers, New Delhi, India

[4] N. Krishna Raju, Advanced Reinforced Concrete Design, CBS Publisher 2015.

[5] IS 456:2000, "Indian Standard Code Of Practice For Plain and Reinforced Concrete", Fourth Revision, Bureau of Indian Standards, New Delhi, July, 2000.

[6] Mitan Kathrotiya, Kaushal Parikh, "Study on Flat Slab and Conventional Slab", International Journal of Scientific Research and Development", Vol. 5, Issue 1, 2017.

[7] S.S.Patil, Rupali Sigi, "Analysis and Design of Flat Slabs Using Various Codes”, Internatiomal Journal of Research in Engineering and Technology", Vol.3, Issue 4, 2014. 